\title{
Electronic structure and ultrafast dynamics of FeAs-based superconductors by angle- and time-resolved photoemission spectroscopy.
}

\author{
I. Avigo', S. Thirupathaiah², E. D. L. Rienks ${ }^{3,4}$, L. Rettig ${ }^{1}$, A. Charnukha ${ }^{5}$, M. Ligges ${ }^{1}$, R. Cortes ${ }^{6}$, J. Nayak $^{7}$, \\ H. S. Jeevan ${ }^{8}$, T. Wolf ${ }^{9}$, Y. Huang ${ }^{10}$, S. Wurmehl ${ }^{3}$, P. Gegenwart ${ }^{8}$, M. S. Golden $^{10}$, B. Büchner ${ }^{3,4}, M^{2}$. Vojta ${ }^{4}$, M. \\ Wolf $^{6}$, C. Felser ${ }^{7}$, J. Fink ${ }^{\star}, 3,4,7$, U. Bovensiepen ${ }^{\star}, 1$
}

${ }^{1}$ Fakultät für Physik, Universität Duisburg-Essen, Lotharstr. 1, D-47057 Duisburg, Germany.

${ }^{2}$ Solid State and Structural Chemistry Unit, Indian Institute of Science, Bangalore, Karnataka, 560012, India.

${ }^{3}$ Institute for Solid State and Materials Research Dresden, Helmholtzstrasse 20, D-01069 Dresden, Germany

${ }^{4}$ Institut für Festkörperphysik, Technische Universität Dresden, D-01062 Dresden, Germany

${ }^{5}$ Department of Physics, University of California, San Diego, La Jolla, California 92093, USA.

${ }^{6}$ Abteilung Physikalische Chemie, Fritz-Haber-Institut der MPG, Faradayweg 4-6, D-14195 Berlin, Germany

${ }^{7}$ Max Planck Institute for Chemical Physics of Solids, D-01187 Dresden, Germany

${ }^{8}$ Institut für Physik, Universität Augsburg, Universitätstr.1, D-86135 Augsburg, Germany

${ }^{9}$ Karlsruhe Institute of Technology, Institut für Festkörperphysik, D-76021 Karlsruhe, Germany.

${ }^{10}$ Van der Waals-Zeeman Institute, University of Amsterdam, NL-1018XE Amsterdam, the Netherlands

Received XXXX, revised XXXX, accepted XXXX

Published online XXXX

Key words: Fe-based superconductors, electronic structure, electron dynamics, ARPES, time-resolved ARPES

* Corresponding author: e-mail uwe.bovensiepen@uni-due.de, Phone +49-203-3794566, Fax +49-203-3794555;

J.Fink@ifw-dresden.de, Phone +49-351-4659425, Fax +49-351-4659313

In this article we review our angle- and time-resolved photoemission studies (ARPES and trARPES) on various ferropnictides. In the ARPES studies we focus first on the band structure as a function of control parameters. We find a Lifshitz transition near optimally "doped" compounds of hole/electron pocket vanishing type. Second we investigated the inelastic scattering rates as a function of the control parameter. In contrast to the heavily discussed quantum critical scenario we find no enhancement of the scattering rate near optimally "doping". Correlation effects which show up by the non-Fermi-liquid behavior of the scattering rates, together with the Lifshitz transition offer a new explanation for the strange normal state properties and suggests an interpolating superconducting state between BardeenCooper-Schrieffer (BCS) and Bose-Einstein (BE) condensation.
Adding femtosecond time resolution to ARPES provides complementary information on electron and lattice dynamics. We report on the response of the chemical potential to coherent optical phonons in combination with incoherent electron and phonon dynamics described by a three temperature heat bath model. We quantify electron phonon coupling in terms of $\lambda\left\langle\omega^{2}\right\rangle$ and show that the analysis of the electron excess energy relaxation is a robust approach. The spin density wave ordering leads to pronounced momentum dependent relaxation dynamics. In the vicinity of $k_{F}$ hot electrons dissipate their energy by electron-phonon coupling with a characteristic time constant of 200 fs. Electrons at the center of the hole pocket exhibit a four times slower relaxation which is explained by spin-dependent dynamics with its smaller relaxation phase space. This finding has implications beyond the material class of Fe-pnictides because it establishes experimental access to spin-dependent dynamics in materials with spin density waves. 
1 Introduction The discovery of high- $T_{c}$ superconductivity in $\mathrm{LaO}_{1-x} \mathrm{~F}_{x} \mathrm{FeAs}$ [1] has started a new era in research on superconductivity [2,3]. In the following years several related families of iron-based superconductors (FeSc's) were discovered. They all have in common that superconductivity is achieved by chemical substitution or pressure starting from antiferromagnetic metallic parent compounds, in which the important components are $\mathrm{FeAs}$ or FeSe/Te layers. Since superconductivity appears at a quantum critical point (QCP) [4, 5, 6] at the end of an antiferromagnetic or nematic region in a phase diagram temperature vs. a control parameter, a wide spread opinion is that superconductivity is mediated by antiferromagnetic or nematic quantum fluctuations to which the charge carriers couple. Related to this is the explanation for the observation of non-Fermi-liquid (NFL) behavior in the normal state above the QCP. Within this scenario, the linear temperature dependence of the resistivity observed in the normal state of FeScs above the quantum critical point near optimal doping can be related to a large coupling of the charge carriers to the antiferromagnetic or nematic quantum fluctuations [7,8]. Furthermore the huge mass enhancement of the charge carriers detected in the London penetration depth measurements near optimal doping was explained by the existence of a quantum critical behavior in this region of the phase diagram [8]. The NFL behavior, which also appears in other unconventional superconductors such as the heavy fermion systems and the cuprates led to the phenomenological theory of the marginal Fermi liquid [9] which describes the many-body properties, i.e., the mass enhancement and the scattering rate, of systems in which the spectral weight of the coherent quasiparticles just disappears due to correlation effects.

Measurements of transport and thermal properties are valuable methods to obtain information on the electronic structure of such kind of solids. An excellent overview on these properties in the field of ferropnictides was recently published [10]. However, such measurements yield information on the properties averaged over the entire Fermi surface. Even in the cuprates, where there is only one band close to the Fermi surface, momentum dependent information is needed since the many-body properties change when moving around the Fermi surface. In the case of the $\mathrm{FeScs}$, there are at least four Fe $3 d$ bands close to the Fermi surface which form hole pockets in the center and electron pockets at the edges of the Brillouin zone (BZ) in this quasi two-dimensional systems. In Fig. 1 we present a schematic drawing of the Fermi surface together with scattering processes.

Since there are very often very strong nesting conditions between hole and electron pockets, the antiferromagnetic and the pairing susceptibility are believed to be dominated by interpocket scattering processes between the hole pockets and the electron pockets. This led to the proposal of s+- superconductivity, in which the superconducting order parameter changes the sign when moving from the hole
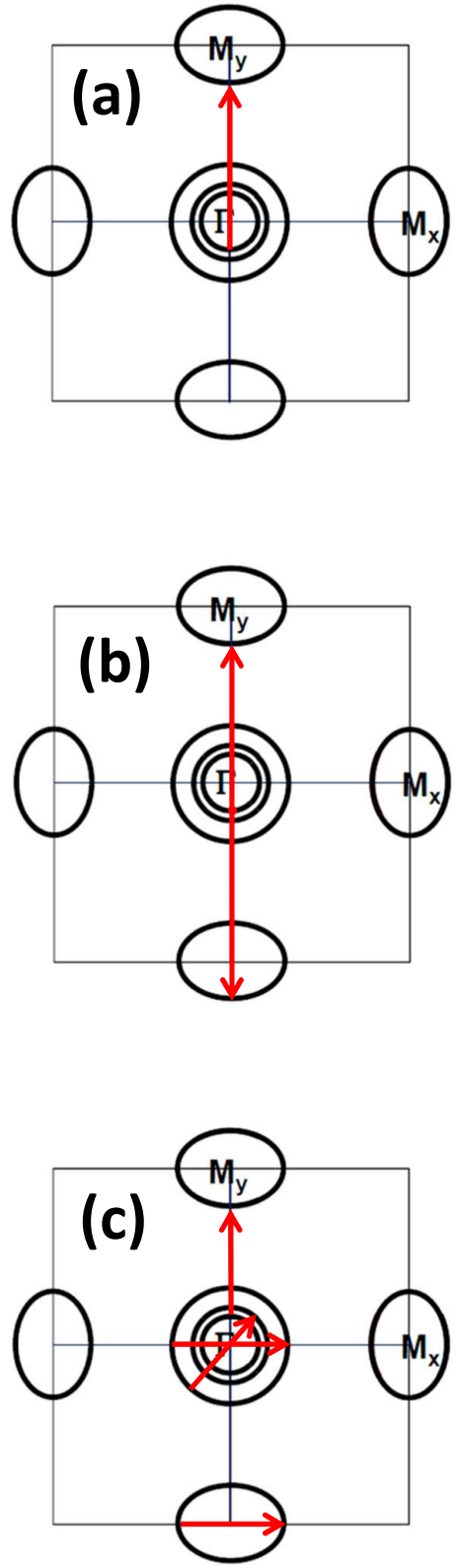

Figure 1 Schematic picture of the quasi two-dimensional Fermi surface of ferropnictides. The red arrows present scattering processes which lead to an antiferromagnetic or nematic susceptibility (a), to a superconducting pairing susceptibility (b), and to transport and thermal properties (c).

pockets to the electron pockets [11]. It is expected that the antiferromagnetic order and the superconducting order are mediated by strong inter- and intra-pocket scattering processes (between hot spots) while the conductivity is related to weak scattering processes (between cold spots). In order 

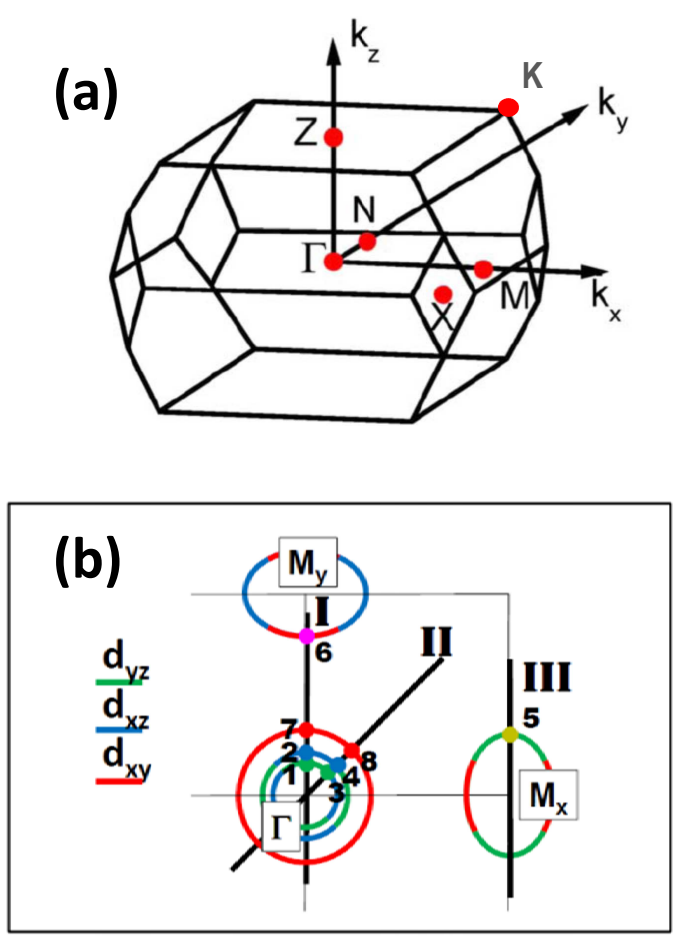

Figure 2 (a) Brillouin zone of ferropnictides. (b) Schematic Fermi surface of ferropnictides in the $k_{z}=$ 0 plane together with cuts performed in the APES and trARPES experiments. The orbital character of the Fermi surfaces was adopted from [13] and is marked in different sections by different colors. Points at which scattering rates were determined are labeled by numbers and different colors.

to obtain a microscopic understanding of superconductivity and the normal state properties (e.g. the conductivity), it is important to obtain information of the scattering process between the various parts of the Fermi surface and even between different sections of the Fermi surfaces. Angleresolved photoemission spectroscopy (ARPES) [12] and time-resolved angle-resolved photoemission spectroscopy (trARPES) are especially valuable methods since they provide momentum-dependent information in the multi-band FeScs for the occupied and unoccupied states, respectively, in the relevant energy range around the Fermi level. In the present review we present measurements near the high symmetry points in various FeScs. This is illustrated in Fig.2, where we show the three-dimensional BZ of ferropnictides together with a cut of the band structure at the Fermi level at $k_{z}=0$. In the ARPES experiments we have performed three cuts (I, II, and III) to reach the points 1-8. In the trARPES experiments we have measured along cut I in the region of the hole pockets.
In the present review we report on results from the compounds $\mathrm{Ba}\left(\mathrm{Fe}_{1-x} \mathrm{Co}_{x}\right)_{2} \mathrm{As}_{2}, \mathrm{BaFe}_{2} \mathrm{As}_{2-x} \mathrm{P}_{x}$, $\mathrm{EuFe}_{2} \mathrm{As}_{2-x} \mathrm{P}_{x}, \mathrm{NaFe}_{1-x} \mathrm{Co}_{x}$ As, and $\mathrm{NaFe}_{1-x} \mathrm{Rh}_{x}$ As. These compounds are usually termed 122 and 111 compounds.

\section{Experimental details}

2.1 Single crystals Using the self-flux method single crystals of $\mathrm{Ba}\left(\mathrm{Fe}_{1-x} \mathrm{Co}_{x}\right)_{2} \mathrm{As}_{2}$ were grown in Amsterdam. Characterization of these samples was reported in [15]. Another set of $\mathrm{Ba}\left(\mathrm{Fe}_{1-x-y} \mathrm{Co}_{x} \mathrm{Mn}_{y}\right)_{2} \mathrm{As}_{2}$ single crystals was grown at the KIT from the self-flux and characterized by resistivity, magnetization, specific heat, and dilatometry measurements [16]. Single crystals of $\mathrm{BaFe}_{2} \mathrm{As}_{2-x} \mathrm{P}_{x}$ and $\mathrm{EuFe}_{2} \mathrm{As}_{2-x} \mathrm{P}_{x}$ were grown in Göttingen and Augsburg using the Sn-flux and the Bridgman (without Sn flux) method, respectively and they were characterized by various methods [17]. Single crystals of $\mathrm{NaFe}_{1-x} \mathrm{Co}_{x} \mathrm{As}, \mathrm{NaFe}_{1-x} \mathrm{Rh}_{x} \mathrm{As}$, and $\mathrm{BaCo}_{2} \mathrm{As}_{2}$ were grown in Dresden using as well the self-flux method [18].

2.2 ARPES ARPES measurements were conducted at the $1^{2}$ and $1^{3}$ ARPES endstations attached to the beamline UE112 PGM 2 at BESSY with energy and angle resolutions between 4 and $10 \mathrm{meV}$ and $0.2^{\circ}$, respectively. Variable photon energies $\mathrm{h} \nu=20-130 \mathrm{eV}$ were used to reach different $k_{z}$ values in the BZ. The use of polarized photons provides information on the orbital character of the electronic states by matrix element effects [20]. If not otherwise stated the measuring temperature at the $1^{2}$ and $1^{3}$ ARPES endstations were 30 and $0.9 \mathrm{~K}$, respectively. Temperatures above the Néel temperature were used for unsubstituted or weakly doped samples in order to keep the compounds in the paramagnetic state and and to avoid back-folding of the bands due to the antiferromagnetic order [21].

Although ARPES is a highly surface sensitive method, previous experimental and theoretical studies using density functional theory (DFT) band structure calculations show that ARPES results are close to the bulk electronic structure. In the 122 compounds a disordered $\mathrm{Ba} / \mathrm{Eu}$ surface layer is formed upon cleaving at low temperatures which leads to additional broadening of the lines due to elastic scattering [22]. Ordering at higher temperatures leads to backfolding of bands. In the 111 compounds, calculations indicate that the electronic structure of the surface layer is close to that of the bulk [23].

2.3 trARPES Femtosecond time- and angle-resolved ARPES measurements reported here were carried out in the Physics Department of the FU Berlin using the experimental setup described in [24,25]. Employing a $300 \mathrm{kHz}$ commercial Ti:sapphire amplifier (Coherent RegA 9050) femtosecond laser pulses at $1.5 \mathrm{eV}$ fundamental photon energy and 55 fs pulse duration were generated. These pulses are used as pump pulses in pump-probe experiments. The probe pulses with photon energy of $6.0 \mathrm{eV}$ are generated by splitting off a fraction of the fundamental pulse which 

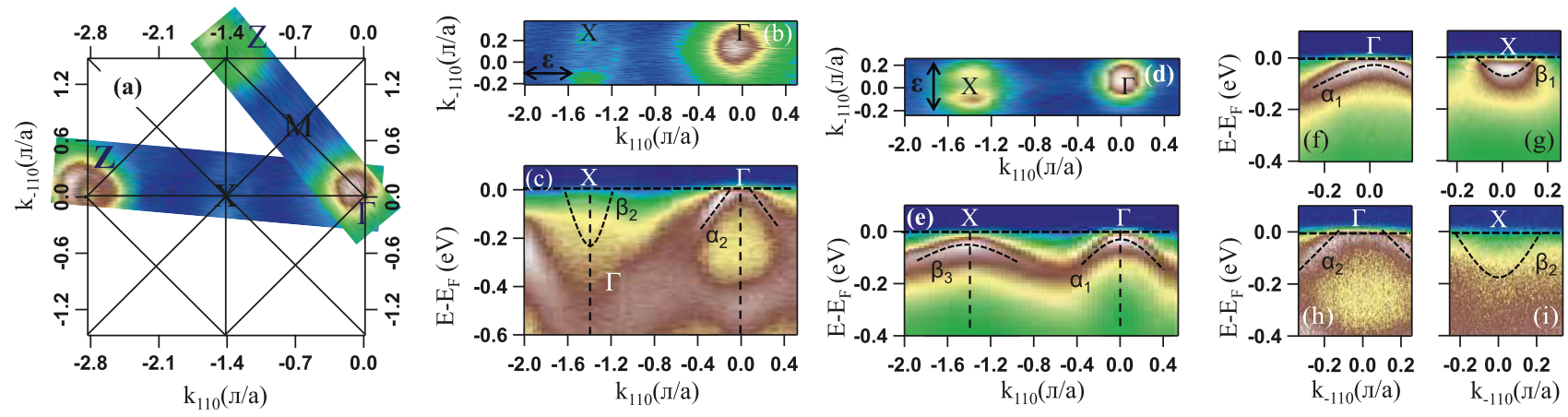

Figure 3 ARPES data of a slightly underdoped $\mathrm{Ba}\left(\mathrm{Fe}_{1-x} \mathrm{Co}_{x}\right)_{2} \mathrm{As}_{2} x=0.04$ single crystal. (a) Fermi surface map taken at the high symmetry points $\Gamma, M, X$ and $Z$ projected on to a tetragonal two dimensional BZ. (b) Fermi surface taken from (a) along $k_{110}$ direction passing through the $X$ and $\Gamma$ points. (c) Energy distribution map (EDM) taken from (b) in order to reveal the band dispersion along the $\Gamma$-X high symmetry line . (d) and (e) Fermi surface map and EDM through the $X$ and $\Gamma$-points, respectively, measured using vertical polarization . (f) and (h) EDMs taken at $\Gamma$ along the $k_{110}$ direction measured using vertical and horizontal polarization, respectively. (g) and (i) EDMs taken at $X$ along the $k_{110}$ direction measured using vertical and horizontal polarization, respectively. Reprint from [14].

is subsequently frequency quadrupled in two subsequent BBO non-linear optical crystals, remaining synchronized with the pump pulses. The obtained sub $100 \mathrm{fs}$ probe pulse duration determines the overall time resolution of the experiment. Temporal and spatial overlap of pump and probe pulses are obtained in ultrahigh vacuum on the sample surface of in-situ cleaved Fe-pnictide single crystals. The energy resolution of the experiment of $50 \mathrm{meV}$ is set by the laser pulse bandwidth required to generate fs laser pulses. To detect the low energy photoelectrons of about $1 \mathrm{eV}$ kinetic energy an electron time-of-flight spectrometer is used in combination with single electron counting. For sample cooling to $25 \mathrm{~K}$ a $1 \mathrm{He}$ cryostat was employed.

\section{ARPES results}

3.1 Band structure, Fermiology, Lifshitz transitions In Fig. 3 we show representative ARPES data of a slightly underdoped $\mathrm{Ba}\left(\mathrm{Fe}_{1-x} \mathrm{Co}_{x}\right)_{2} \mathrm{As}_{2} x=0.04$ single crystal [26, 19]. Fermi surfaces were derived from an integration of the spectral weight close to the Fermi level. In the energy distribution maps (EDMs) we see - depending on the polarization - the inner hole pocket $\left(\alpha_{1}\right)$ or the middle hole pocket $\left(\alpha_{2}\right)$ near $\Gamma$. The intensity of the outer hole pocket with predominantly $3 d x y$ orbital character is very weak in this compound because bands with this orbital character exhibit the highest elastic scattering rates [27]. In addition, electron pockets $\left(\beta_{1}\right.$ and $\left.\beta_{2}\right)$ are detected near $X$.

In Fig. 4 we present photon energy dependent data measured near the $\Gamma$ and the $X$ point which provide information on the $k_{z}$ dispersion of the bands [19]. In agreement with density functional theory (DFT) band structure calculation [19], a modest doping dependent $k_{z}$ dispersion is detected in the ARPES data for the hole pockets and a very weak one for the electron pockets.
It is interesting to look closer at the doping dependent band structure. As presented in Fig. 4 the size of the Fermi surface in the $k_{x}-k_{y}$ plane at $\Gamma$ decreases rapidly with increasing Co concentration while the reduction at the $Z$ point is weaker. This result is also obtained in a more refined evaluation of the $k_{F}$ values shown in Fig. 5] [19]. In particular at the $\Gamma$ point the $k_{F}$ values shown in Fig. 3 show a strong concentration dependence. There the top of the inner hole pocket moves through the Fermi level near optimal doping $(x=0.06)$, i.e., where the Fermi wave vector goes to zero (Fig. 5 panel $\Gamma$ ) and where the Fermi surface disappears (Fig. 4 (d) and (g)). Thus for the inner hole pocket there is a Lifshitz transition of the pocket vanishing type [28] at the $\Gamma$ point: the Fermi cylinder transforms into an ellipsoid around the $Z$ point (see Fig. 6). As a consequence of the Lifshitz transition, there are flat bands at the Fermi level. In the overdoped region, the length of the ellipsoid along the $k_{z}$ line is getting shorter and shorter and finally at the end of the superconducting region the inner hole pocket becomes completely filled also at the $Z$ point (see Fig.6).

In Fig.7 we plot the top of the inner hole hole pocket and the bottom of the electron pocket having predominantly both $y z$ character at the points 1 and 5 in Fig.2.(b) as a function of $3 d$ count, i.e., the number of $3 d$ electrons in the transition metal ion. We focus to these points since at these points the largest superconducting gaps have been detected together with the largest scattering rates (see below). For the electron doped ferropnictides the top of the inner hole pocket moves through the Fermi level very close to the optimal doping concentration, while for the hole doped systems the bottom of the electron pocket moves through the Fermi level at optimal doping. Thus we have a Lifshitz transition of bands having $y z$ character in both cases. 

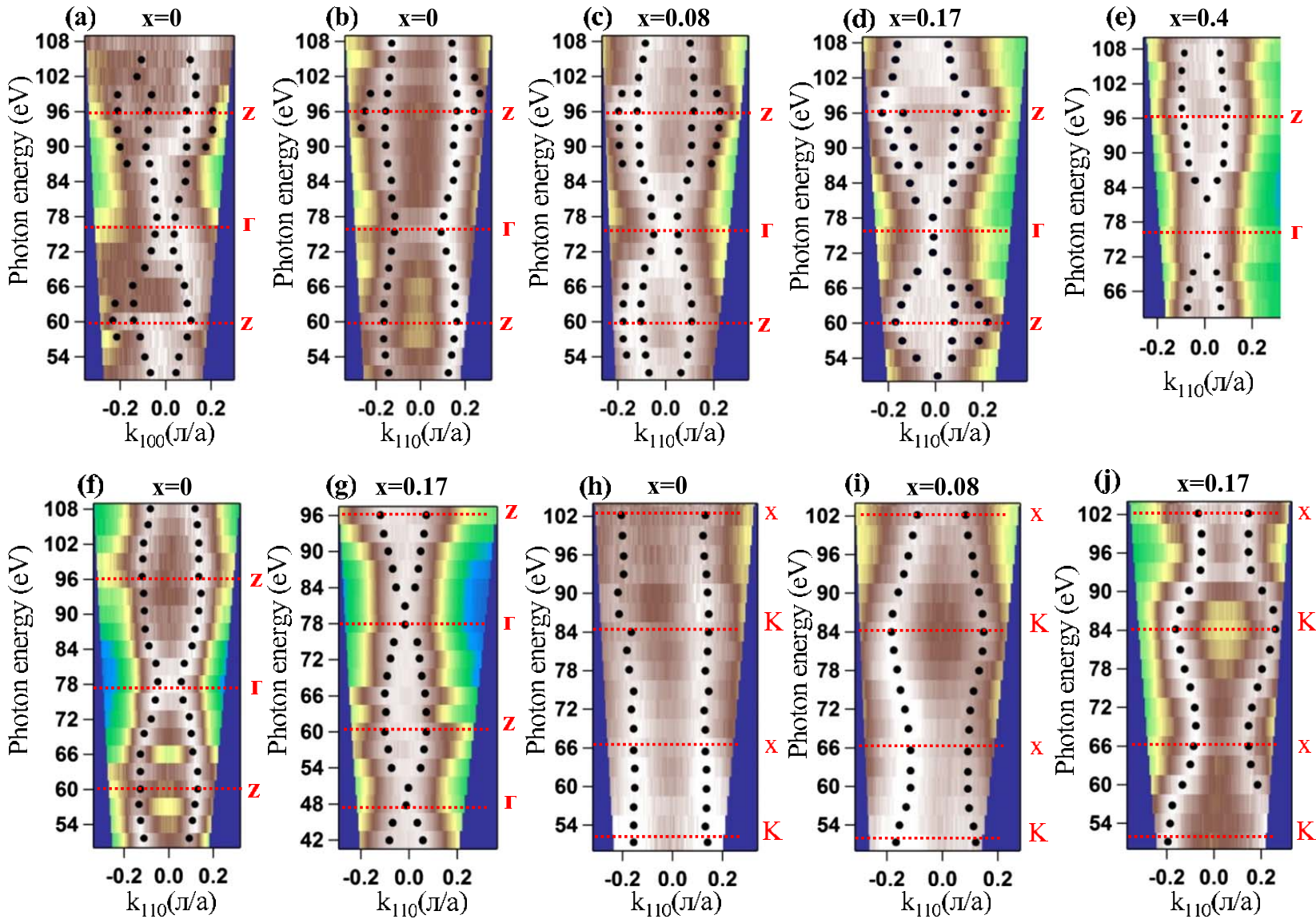

(h) $\mathbf{x}=\mathbf{0}$
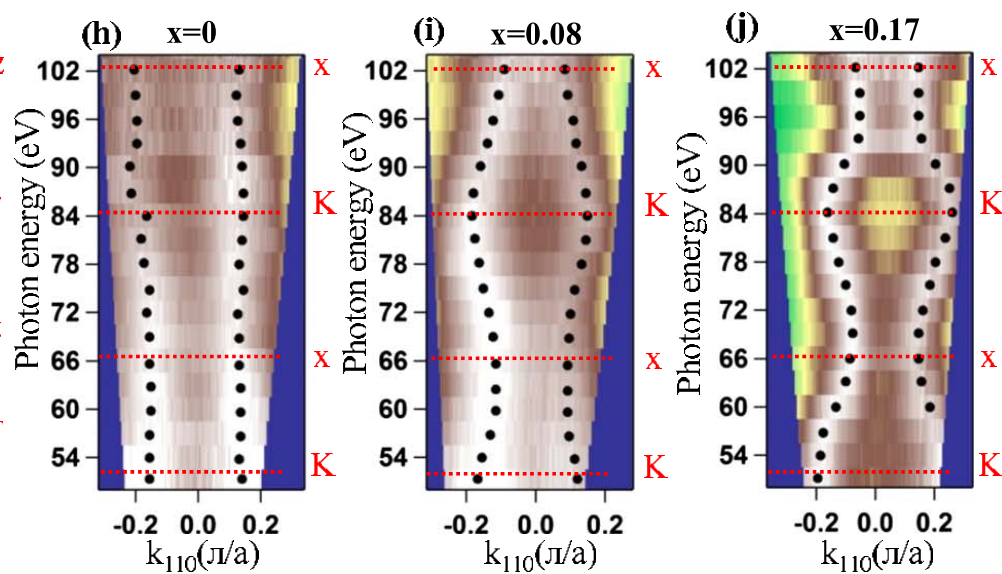

Figure 4 Photon energy dependent ARPES measurements performed on $\mathrm{Ba}\left(\mathrm{Fe}_{1-x} \mathrm{Co}_{x}\right)_{2} \mathrm{As}_{2}$ to reveal the $k_{z}$ dispersion as a function of doping concentration. (a) Fermi surface for $x=0$, in the $k_{100} \mathrm{vs} \mathrm{h} \nu$ plane near $k_{x}=k_{y}=0$ measured with p-polarized photons. (b) Analogous data as in (a) but in the $k_{110} \mathrm{vs} h \nu$ plane. (c), (d), and (e) Analogous data as in (b) but for $\mathrm{x}=0.8,0.17$, and 0.4 , respectively. (f) and (g) Analogous data as in (b) but measured with s-polarized photons for $x=0$ and 0.17. (h), (i), and (j) Fermi surfaces in the $k_{110}$ vs h $\nu$ plane near $k_{x}=k_{y}=1$ measured with s-polarized photons for $x=0,0.08$, and 0.17, respectively. Reprint from [19]. Copyright 2010, American Physical Society.

Very similar data were derived for the isovalently substituted compounds $\mathrm{BaFe}_{2} \mathrm{As}_{2-x} \mathrm{P}_{x}$ and $\mathrm{EuFe}_{2} \mathrm{As}_{2-x} \mathrm{P}_{x}$ [26]. Also in these systems a Lifshitz transition of the inner hole pocket is observed near optimal substitution. Different from the electron doped compound is that in order to achieve charge neutrality, the Fermi surface at the $Z$ point increases (see Fig. 6). Thus even at the highest $\mathrm{P}$ concentration there is still a partially empty hole pocket having $x z / y z$ character. Possibly this observation is related to the fact that superconductivity is observed even in the pure $\mathrm{BaFe}_{2} \mathrm{P}_{2}$ compound.

3.2 Scattering rates In this section we review our ARPES results on the scattering rates in ferropnictides. In Fig. 8(a) and (d) we show typical energy distribution maps (MDCs) of optimally doped/substituted $\mathrm{BaFe}_{2} \mathrm{As}_{2-x} \mathrm{P}_{x}$ and $\mathrm{NaFe}_{1-x} \mathrm{Rh}_{x} \mathrm{As}$. In (a) the inner two hole pockets and in (d) all three hole pockets can be clearly resolved.
Typical momentum cuts (MDCs) together with fits by Lorentzians are shown in Fig. 8. b) and (e). In Fig. 8.c) and (f) we present the scattering rates $\Gamma(E)$ derived from the width in momentum space [29]. For the hole pockets in $\mathrm{BaFe}_{2} \mathrm{As}_{2-x} \mathrm{P}_{x}$ we find in a large energy range of $5 \leq E \leq 120 \mathrm{meV}$ a NFL linear-in-energy dependence, which can be described by $\Gamma(E)=\alpha+\beta E$. Due to a finite energy and momentum resolution $5 \mathrm{meV}$ is the lower boarder of the range in which reasonably values for the scattering rates can be derived in the present ARPES experiments. Similar results were obtained for $\mathrm{Ba}\left(\mathrm{Fe}_{1-x} \mathrm{Co}_{x}\right){ }_{2} \mathrm{As}_{2}$ and $\mathrm{Ba}\left(\mathrm{Fe}_{1-x-y} \mathrm{Co}_{x} \mathrm{Mn}_{y}\right)_{2} \mathrm{As}_{2}$ [30]. Since in $\mathrm{NaFe}_{1-x} \mathrm{Rh}_{x}$ As there is a strong hybridization between bands close to the Fermi level, the energy ranges for which the scattering rates can be determined, is restricted. $\alpha$ is a measure for elastic scattering processes, e.g. scattering by impurities. $\beta$ is a measure of the 


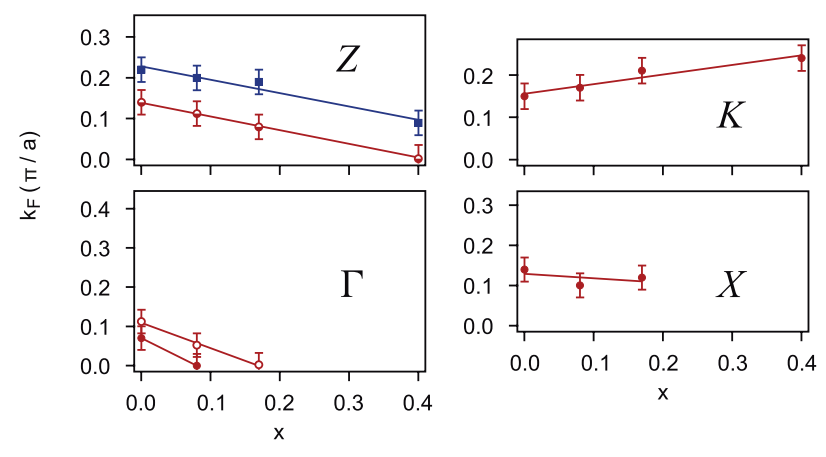

Figure 5 Fermi vectors of $\mathrm{Ba}\left(\mathrm{Fe}_{1-x} \mathrm{Co}_{x}\right)_{2} \mathrm{As}_{2}$ as a function of Co doping near the high symmetry points $Z, \Gamma, K$, and $X$. The orbital character of different bands is marked by different symbols: open (closed) circles predominantly $x y(x z / y z)$ character, closed squares predominantly $z^{2}$ character. Reproduced from [26]

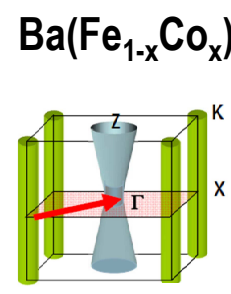

ud

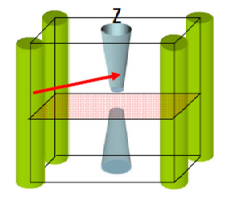

op

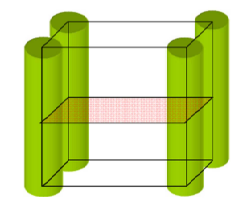

od

\section{$\mathrm{Ba} / \mathrm{EuFe}_{2-\mathrm{x}} \mathrm{As}_{2-\mathrm{x}} \mathrm{P}_{\mathrm{x}}$}

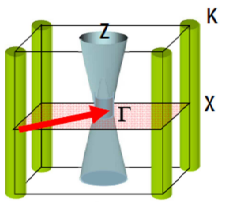

ud

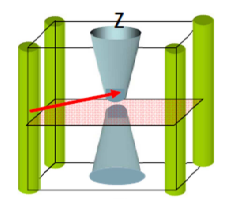

op

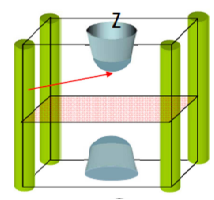

od
Figure 6 Schematic illustration of the Fermi cylinders of the $x z / y z$ hole (blue) and electron pockets (green) in $\mathrm{BaFe}_{2} \mathrm{As}_{2}$ as function of electron doping by Co substitution of $\mathrm{Fe}$ (upper panel) and chemically pressurizing by isovalent $\mathrm{P}$ substitution of As (lower panel). ud: undoped, op: optimaly doped/substituted, od: overdoped/oversubstituted.

strength of inelastic scattering processes. In the inelastic scattering rates or the imaginary part of the self-energy no evidence for a strong coupling to bosonic excitations such as phonons is detected. If phonons would determine the scattering rate a step like increase should be observed at the phonon energies (which are smaller than $40 \mathrm{meV}$ ) [31].

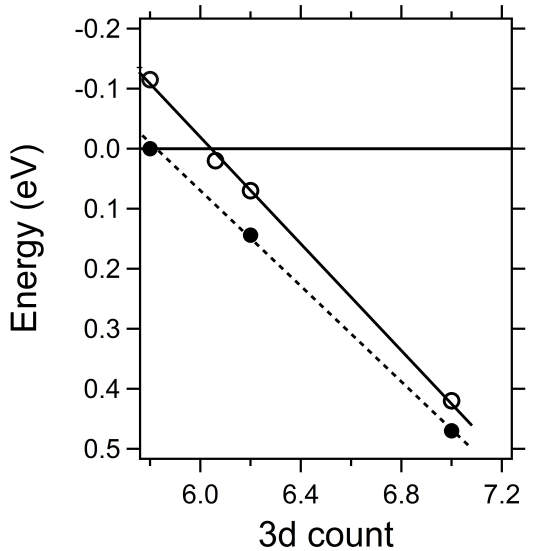

Figure 7 The top of the inner hole pocket in $\mathrm{Ba}\left(\mathrm{Fe}_{1-x} \mathrm{Co}_{x}\right)_{2} \mathrm{As}_{2}$ compounds (open circles) and the bottom of the electron pocket (closed circles) as a function of $3 d$ count. Data from nearly optimally hole doped $\mathrm{K}_{x} \mathrm{Ba}_{1-x} \mathrm{Fe}_{2} \mathrm{As}_{2}$ are added.

Moreover, in the present ARPES data no kinks in the dispersion are realized which are expected when the charge carriers are strongly coupled to phonons [12]. Thus the observed energy dependence clearly shows that the scattering rates are predominantly determined by electronic excitations, i.e., by an Auger process in the valence band: a relaxation of the photo-electron hole to lower binding energies and a transfer of the received energy to an electron-hole excitation [32].

Differences in the inelastic scattering rates have been predicted by theoretical calculations [33, 13]: scattering rates between sections of the Fermi surface having the same orbital character (e.g. from point 1 to point 5 in Fig.2(b) having both predominantly $y z$ orbital character) should be bigger than scattering rates between sections having a non-equal orbital character (e.g. from point 2 to point 5 in Fig. 2(b) having predominantly $x z$ and $y z$ orbital character, respectively). The reason for this is that the former is determined by the on-site Coulomb repulsion $U$, while the latter is reduced to $U-J$ due to the Hund's exchange coupling $J$ which appears when considering transitions between parallel spin states. Parallel spins should occur in the case when the orbital character of the initial and the final state in the scattering process are antisymmetric.

The linear dependence of the scattering rates, i.e., NFL behavior, indicate a considerable amount of correlation effects in the Fe $3 d$ system. Such a behavior is often described by the phenomenological model of a marginal Fermi liquid [9].

In Fig.9 a compilation of all available $\beta$ values for hole and electron pockets in $\mathrm{BaFe}_{2} \mathrm{As}_{2-x} \mathrm{P}_{x}$ and $\mathrm{EuFe}_{2} \mathrm{As}_{2-x} \mathrm{P}_{x}$, in $\mathrm{NaFe}_{1-x} \mathrm{Co}_{x}$ As and $\mathrm{NaFe}_{1-x} \mathrm{Rh}_{x} \mathrm{As}$ are presented. Interestingly no enhancement of the scattering rate is observed near optimal doping. This is in stark 

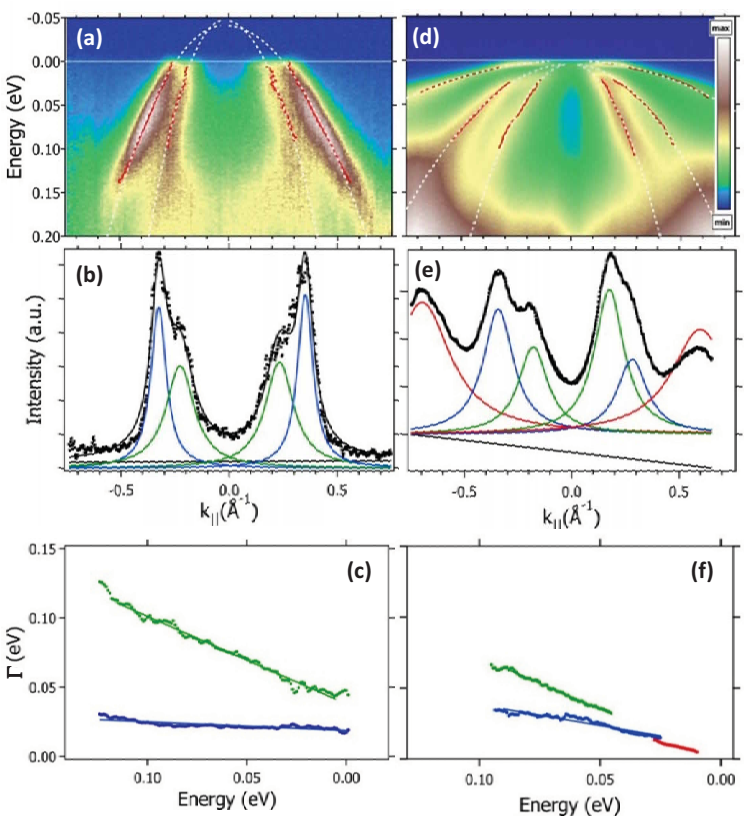

Figure 8 (a) and (d) ARPES energy distribution maps of $\mathrm{BaFe}_{2} \mathrm{As}_{2-x} \mathrm{P}_{x} x=0.27$ and $\mathrm{NaFe}_{1-x} \mathrm{Rh}_{x}$ As $x=0.027$ , respectively, measured along cut I. The red lines depict dispersions derived from the momentum distribution curves, which are shown in (b) and (e) for a binding energy of $40 \mathrm{meV}$. (c) and (f) Experimental scattering rates $\Gamma(E)$ for $\mathrm{BaFe}_{2} \mathrm{As}_{2-x} \mathrm{P}_{x} x=0.27$ and $\mathrm{NaFe}_{1-x} \mathrm{Rh}_{x} \mathrm{As}$ $x=0.027$, respectively, together with a linear fit. Green: near point 1 , blue: near point 2 , red: near point 7 (see Fig. 2(b)) [29].

contrast to what is naively expected in the quantum critical scenario: the scattering rates should be strongly enhanced near the QCP due to a coupling to antiferromagnetic or nematic quantum fluctuations.

3.3 Coaction of Lifshitz transitions and correlation effects. Recently an explanation of the appearance of a linear-in-energy increase of the scattering rate was presented pointing out the importance of a Lifshitz transition [34]. A simple calculation of the $\Im \Sigma$, in which a constant density of states at the Fermi level was assumed, as a function of the extension of the unoccupied part of the band above $E_{F}$ yielded a NFL behavior near the Lifshitz transition, i.e., when the the top of the hole pocket $\left(E_{t}\right)$ is close to the Fermi level. Decreasing $E_{t}$, i.e., increasing the energy difference $E_{F}-E_{t}$ and thus moving away from the Lifshitz transition, extends the range of Fermi liquid behavior to higher energies. On the other hand, a decrease of an $\left|E_{F}-E_{t}\right|$ or an increase of correlation effects which renormalizes the width of unoccupied states and thus reduces also $\left|E_{F}-E_{t}\right|$, moves the NFL behavior to lower energies. If $E_{t}$ is related to a control parameter and correlation effects are important, this means that NFL behavior
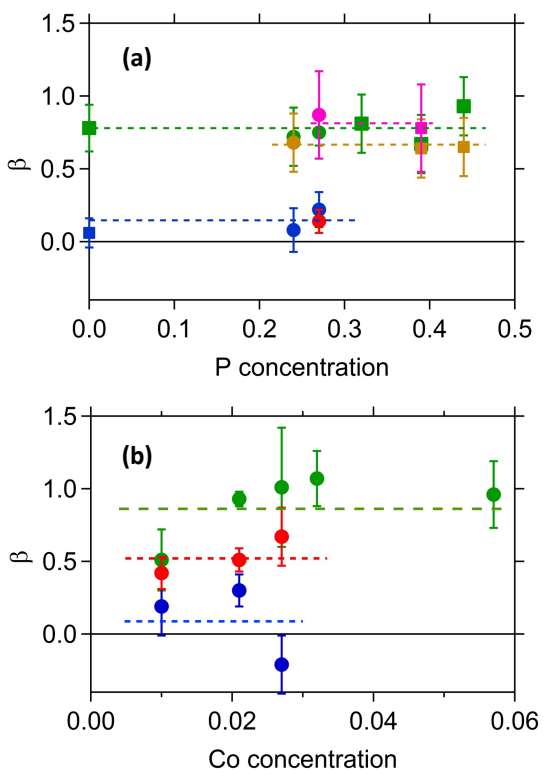

Figure 9 Doping/substitution dependent $\beta$ values in $\mathrm{BaFe}_{2} \mathrm{As}_{2-x} \mathrm{P}_{x}$ and $\mathrm{EuFe}_{2} \mathrm{As}_{2-x} \mathrm{P}_{x}$ (upper panel), and $\mathrm{NaFe}_{1-x} \mathrm{Co}_{x}$ As and $\mathrm{NaFe}_{1-x} \mathrm{Rh}_{x}$ As ( lower panel) for various high-symmetry points near $\Gamma$ and $X$. The color code of Fig. 2(b) for the high-symmetry points is used. Reproduced from [29]

should appear at lower energies and in a wider range of the control parameter. This is a consequence of a constant susceptibility for electron-hole excitation which follows from a convolution of a delta-function like unoccupied density of states with a constant occupied density of states.

This idea was supported by further evaluation of our ARPES data. As shown above, from these data we could derive $\Im \Sigma$. Since this function is connected to $\Re \Sigma$ by the Kramers-Kronig relation, we could calculate the latter from our ARPES data. In these calculations the experimental $\Im \Sigma$ was extrapolated linearly to zero energy and at high energy a cutoff energy of $E_{c}=1.5 \mathrm{eV}$ was introduced. Alternatively, we used the parameters which describe $\Im \Sigma$ and use the marginal Fermi liquid model to calculate $\Re \Sigma$. In this model the self-energy is given by [9]

$$
\Sigma=\frac{1}{2} \lambda_{M F} E \ln \frac{E_{c}}{E}-i \frac{\pi}{2} \lambda_{M F} E .
$$

In order to obtain representative results for the FeScs we used in the calculations presented in Fig. 10 a coupling constant of $\lambda_{M F}=1.4$. This value is between those derived for the hole doped and the electron doped compounds [35]. The renormalized dispersion is derived by a subtraction of the calculated $\Re \Sigma$ from the bare particle dispersion derived from DFT band structure calculations. In Fig. 10 we present such calculations as a function of three values of $E_{F}-E_{t}$, simulating underdoped (upper row), 

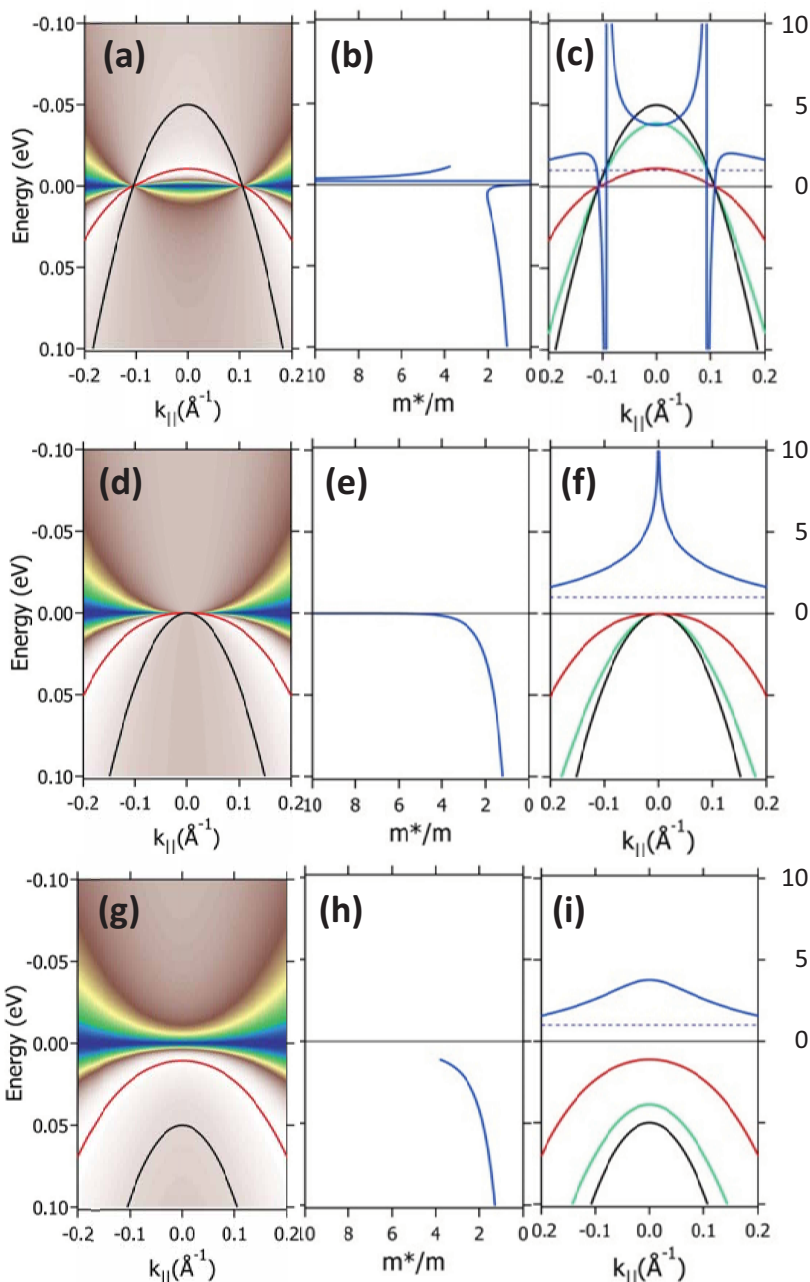

Figure 10 Calculation of electronic structure data for FeScs. Left column: false color map of the spectral function together with the bare particle dispersion (black) and the renormalized dispersion (red). Middle column: energy dependent effective mass $m^{*} / m_{0}$ (blue). Right column: bare particle dispersion (black), $\Re \Sigma$ (green) and renormalized dispersion (red). Top row: $E_{t}=-50 \mathrm{meV}$, simulating underdoped compounds; middle row $E_{t}=0$, simulating optimally doped compounds close to a Lifshitz transition; bottom row: $E_{t}=-50 \mathrm{meV}$, simulating overdoped compounds.

optimally doped (middle row), and overdoped (lower row) compounds. In the left column a calculation of the spectral function is presented. In the middle column the derived effective mass $m^{*} / m_{0}$ as a function of the energy is depicted. In the right column we show the bare particle dispersion (black), the $\Re \Sigma$ (green), and the renormalized dispersion (red). In addition we show in the right panels the effective mass $m^{*} / m_{0}$ by a blue line, as a function of momentum. For $E_{t}=E_{F}$, where the top of the bare particle dispersion just touches the Fermi level, i.e., when at the Fermi level the dispersion of the bare particle band is flat (see Fig. 10(f)), the $\Re \Sigma$ is very close to the bare particle band and therefore the renormalized dispersion at the
* Fermi level is strongly reduced. This corresponds to a very high effective mass $m^{*} / m_{0} \approx 8$ at $k=0$ (see Fig. 10(f)). In Fig. 10.e)), a similar mass enhancement appears close to the Fermi level. The spectral function (Fig. 10 (d)) is dominated by incoherent spectral weight, i.e., by particles for which the life-time broadening is larger than the binding energy. Interestingly, a large completely incoherent mirror like spectral weight is observed as a satellite structure above the Fermi level. Together with a weak quasiparticle structure below the Fermi level, the incoherent spectral \& weight below and above $E_{F}$ allows strong intra pocket and * inter pocket scattering with electron pockets which leads to large low-energy scattering rates which are characteristic for marginal Fermi liquids and which leads to the strong mass enhancement at low energies. Moving away from $k=0$ or $E=E_{F}$, the effective mass is strongly reduced to a value of $m^{*} / m_{0} \approx 2$.

Changing $E_{t}$ to $-50 \mathrm{meV}$ or $50 \mathrm{meV}$, corresponding to a change of the control parameter to underdoped and overdoped compounds, respectively, strongly reduces the size of the effective mass (see Fig. 10 (a), (b), (e), and (f)). छ As expected the incoherent spectral weight far above the $\stackrel{*}{*}$ Fermi level is not changing very much as a function of the control parameter.

A combination of the band structure results and the calculation of the effective mass on the basis of experimental scattering rates leads to the following explanation of the NFL behavior revealed in the ARPES experiments. Using the $3 d$ count dependence of $E_{t}$ leads in $\mathrm{Ba}\left(\mathrm{Fe}_{1-x} \mathrm{Co}_{x}\right)_{2} \mathrm{As}_{2}$ to a concentration dependence $E_{t}=$ $0.4 x$. This would lead to a NFL behavior above our resolution determined low energy boarder of $5 \mathrm{meV}$ above the energy $|0.4(x-0.06)|$ which would lead to a NFL behavior in a narrow concentration range of $x=0.06 \pm 0.0125$. On the other hand, a low energy mass enhancement of eight would result in a NFL behavior in a much larger concentration range of about $x=0.06 \pm 0.1$. This explains our ARPES results of a NFL behavior above $5 \mathrm{meV}$ in a large concentration range.

Summarizing the results of the calculations based on the results of the ARPES experiments, at the Lifshitz transition and only at this transition one obtains a correlationinduced particularly enhanced flattening of the renormalized band which is related to an almost diverging mass at the Fermi level and at the momentum where the Lifshitz transition occurs. Moreover, the correlation effects also lead to a pinning of the top of the hole pocket at the Fermi level.

Finally a calculation of the effective mass as a function of temperature or the thermal energy $k_{\mathrm{B}} T$ and the shift of the top of the hole pocket $E_{t}$ is presented in Fig. 11. An enhancement of the effective mass near the Lifshitz transition and when moving away from the Lifshitz transition a reduction at low temperatures is realized.

The presented calculations offer a new explanation of the NFL behavior of ferropnictides near the QCP. This ex- 


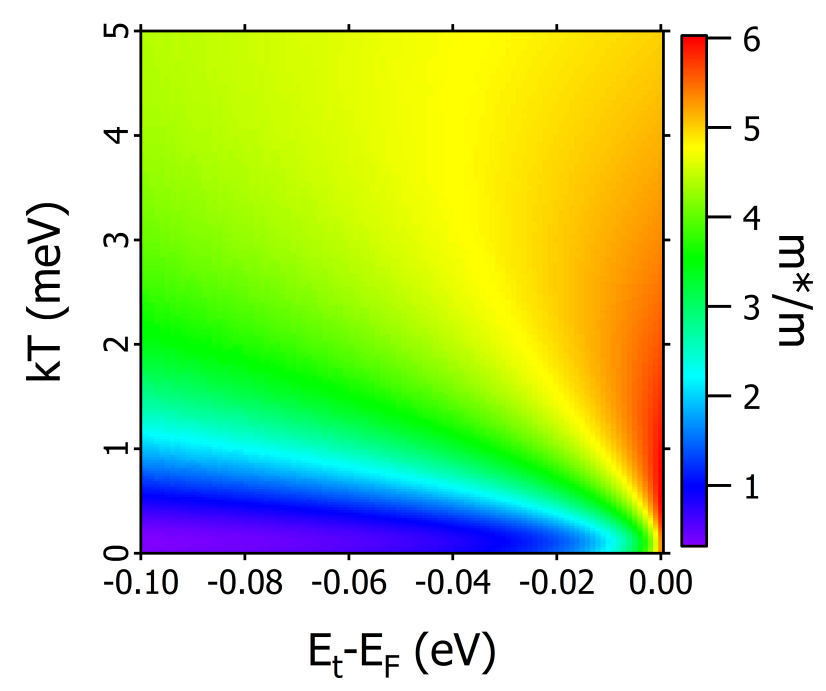

Figure 11 Calculation of the effective mass as a function of the thermal energy $k_{B} T$ and the shift of the top of the hole band $E_{t}$. Reprint from [34].

planation is different from the traditional view which is related to a coupling of the charge carriers to quantum fluctuations at the end of the antiferromagnetic or nematic range. The presented scenario is able to explain the large mass enhancement near optimal substitution in $\mathrm{BaFe}_{2} \mathrm{As}_{2-x} \mathrm{P}_{x}$ derived from the London penetration depth [36, 37] and the de Haas-van-Alphen effect measurements [38] near the QCP. Moreover, the divergent thermopower detected in $\mathrm{Ba}\left(\mathrm{Fe}_{1-x} \mathrm{Co}_{x}\right)_{2} \mathrm{As}_{2}$ near optimal doping [39] and the thermal properties [40] can be explained within the presented scenario.

The discussion of the importance of weakly dispersing bands at the Fermi level for superconductivity dates back to the explanation of high- $T_{c}$ superconductivity in alloys with A15 structure (e.g. $\mathrm{V}_{3} \mathrm{Si}$ ) by a peaked density of states at the Fermi level $N\left(E_{F}\right)$ caused by a quasi-one-dimensional single particle electronic structure [41]. Later on, a correlation of the transition temperatures with Fermi temperatures, derived from magnetic-field penetration depth measurements, showed that in particular the unconventional superconductors have an effective Fermi energy close to to the pair binding energy [42]. This indicates that in the high- $T_{c}$ superconductors a breakdown of the Migdal theorem [43] occurs which was the basis of the standard BCS theory of superconductivity [44] and which requires the inclusion of non-adiabatic effects and the generalization of the Eliashberg equations [45] and which interpolates between the BCS theory and the Bose-Einstein condensation. Moreover, a recent compilation of the electronic structure of unconventional superconductors [46] pointed out that most of the high- $T_{c}$ superconductors have a van Hove sin- gularity at the Fermi level. On the other hand, many of the mentioned compounds fulfill this criteria but have no peaked $N\left(E_{F}\right)$. For example the optimally electron-doped ferropnictides have a van Hove singularity, i.e., an edge at the chemical potential $\mu$ but all DFT calculations predict no peaked bare band density of states at $\mu$ in this quasitwo-dimensional electronic structure. On the other hand the co-action of a Lifshitz transition and correlation effects described in this review leads to a small effective Fermi energy and a high $N\left(E_{F}\right)$. This can lead to an interpolating superconducting state between BCS and BE condensation [47]. The results can be generalized to other unconventional superconductors and are possibly a recipe for future search of high- $T_{c}$ superconductors.

4 Ultrafast dynamics in Fe-122 investigated by femtosecond time-resolved ARPES Ultrafast pumpprobe experiments investigate the response of a material after an optical excitation by a laser pulse. On the probed femtosecond time scales elementary scattering processes are analyzed and provide insight into electron dynamics in the time domain [48], complementary to the line width analysis of static ARPES in the frequency domain. Note, however, that the dynamics probed by ARPES and trARPES are fundamentally different. While in static ARPES a single photo hole is analyzed, trARPES investigates the hot quasiparticle population dynamics in response to an optical excitation which might involve in particular close to the Fermi level several quasiparticle excitations which may interact [49]. Therefore, these two experimental approaches are highly complementary to each other.

Femtosecond time-resolved experiments of complex materials like charge density wave compounds and high temperature superconductors started in the late 1990's by detecting the change of the optical reflectivity at the Ti:sapphire laser's fundamental photon energy of $1.5 \mathrm{eV}$ and at few $\mathrm{THz}$ frequency [50]. At about the same period time-resolved photoemission experiments started to be performed in order to measure hot electron life times several eV above the Fermi level [51]. Few years later relaxation of the hot electron distribution was analyzed for $\mathrm{Bi}_{2} \mathrm{Sr}_{2} \mathrm{CaCu}_{2} \mathrm{O}_{8+\delta}$ by trARPES in order to determine the e-ph coupling [52] and since then this rather young technique was developed step by step [53].

As sketched in Fig. 12(a) trARPES is essentially a pump-probe experiment which analyzes as a function of time delay between pump and probe laser pulses the cross correlation of the spectral weight of optically excited quasiparticles and their subsequent relaxation due to interaction with further fermionic and bosonic excitations. Note that in the data reported here probing in linear photoemission was employed, which gives rise to a time-independent contribution to the detected photoemission intensity. The use of laser pulses in the ultraviolet (UV) spectral range has the advantage of a rather efficient generation processes 

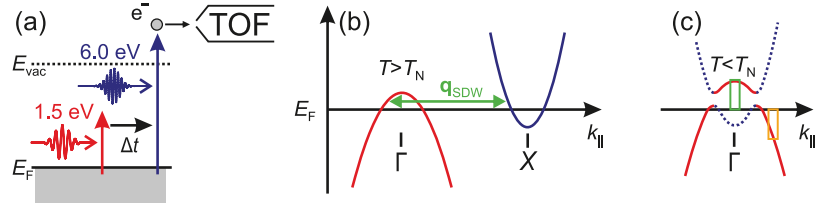

Figure 12 (a) Schematic representation of femtosecond time-resolved ARPES. Femtosecond laser pulses of photon energy 1.5 (pump) and $6.0 \mathrm{eV}$ (probe) are spatially and temporally overlapped on the Fe-pnictide surface. Emitted photoelectrons are detected by an electron time-of flight spectrometer and analyzed as a function of time delay $\Delta t$ between the pump and probe pulses. (b) Simplified sketch of the Fe-122 electronic structure above the Néel temperature $T_{\mathrm{N}}$ with hole and electron pockets. (c) Backfolding of the electron pocket onto $\Gamma$ and interaction with the hole pocket due to formation of antiferromagnetic order below $T_{\mathrm{N}}$. Reprinted with permission from [54]. Copyright 2012 by the American Physical Society.

and allows to detect a high dynamic range of the pumpinduced spectral changes. In the present case two times the second harmonic was generated subsequently in non-linear optical crystals which gives $6.0 \mathrm{eV}$ laser pulses for probing. The low kinetic energy of the photoelectrons limits the accessible parallel momentum component of the photoelectron $\hbar k_{||}$to the center of the first Brillouin zone close to $\Gamma$ with an extension of several $0.1 \AA^{-1}$. Setups employing higher photon energy in the VUV or XUV range probe the full first or further Brillouin zones but the number of detected photoelectrons is usually lower, due to a less efficient and more complex generation process employing higher harmonic generation [53].

Considering the electron and hole pockets of the Fepnictide electronic structure at the BZ boundary and the $\Gamma$-point, electrons of the hole pocket or those which were scattered into the region around the $\Gamma$-point, e.g, by $q_{\mathrm{SDW}}$ are detected, see Fig. 12(b,c). Note, that in the present case of Fe-122 this results in a finite $k_{z} \approx 2 \AA^{-1}$, corresponding to $\approx 0.6(Z-\Gamma)$, at which we detect photoelectrons. Comparing to Fig. 4 this implies that we detect a part of the hole pocket in particular for optimal doping. The results reported here analyze the response of the electron distribution function and the chemical potential to the pump excitation, e-ph coupling, and a spin-dependent contribution to the relaxation dynamics which was determined in the AFM (or SDW) ordered state.

4.1 Response of the electron distribution function and the chemical potential to an optical excitation Absorption of the pump pulse in the investigated material results initially in Drude and / or interband electronic excitations exhibiting a non-equilibrium electron distribution function. Subsequently, scattering with further electrons and bosonic excitations changes the distribution func-

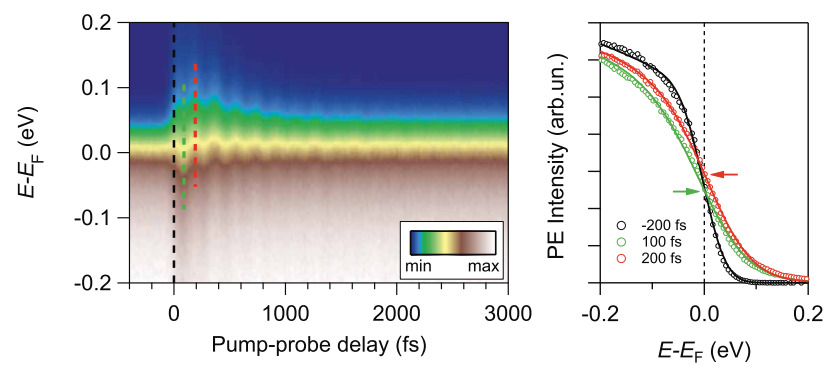

Figure 13 (a) Color coded intensity plot of the trARPES intensity of $\mathrm{BaFe}_{1.85} \mathrm{Co}_{0.15} \mathrm{As}_{2}$ at the $\Gamma$ point as a function of binding energy and pump-probe delay, taken with an incident pump fluence of $F=1.4 \mathrm{~mJ} \mathrm{~cm}^{-2}$ at $T=100 \mathrm{~K}$. The red and green dashed lines mark the spectra shown in (b). (b) trARPES spectra for the first minimum (green) and maximum (red) of the oscillation in comparison to a spectrum before excitation (black). The solid lines are fits to the data. The Fermi level position, $E_{\mathrm{F}}$, extracted from the fit is indicated by the red and green arrows. It is oscillating due to the coherent phonon excitation. Reproduced with permission from [55]. Copyright IOP Publishing. All rights reserved.

tion which eventually may be described by a thermalized distribution function at increased temperatures compared to the situation before pumping [24,53]. The material can respond to the pump excitation in addition by a change in the electronic structure.

Fig. 13 shows at left the time-dependent photoelectron intensity in a false color plot around $E_{\mathrm{F}}$. Upon optical excitation at $t=0$ the intensity distribution broadens by about $\pm 100 \mathrm{meV}$ and exhibits a well defined periodic variation of the high energy cutoff of the spectrum. We conclude from this observation that the pump excitation gives rise to incoherent and coherent excitations. The incoherent ones relax within 1-2 ps and represent electronic excitations. Note that although the pump photon energy is $1.5 \mathrm{eV}$ the largest pump-induced spectral changes occur at much smaller energy which indicates that the primarily excited carriers have scattered inelastically before being detected. The right panel of Fig. 13 depicts three spectra taken from the data set shown at left at the time delays indicated. The spectra after the pump excitation at positive time delays are broadened similar to an increased electronic temperature. We will come back to this aspect in Sec. 4.2 and analyze the energy content in the excited system. The coherent spectral redistribution occurs with a well defined frequency of $5.5 \mathrm{THz}$ which can be assigned to the Raman active $\mathrm{A}_{1 \mathrm{~g}}$ phonon mode corresponding to a displacement of the As atoms perpendicular to the FeAs layers. For details of the frequency determination see Refs. [56, 57]. A detailed analysis revealed further modes to contribute [56]. Why do such coherent phonons modify the high energy cutoff of the photoemission spectrum? Further experiments per- 
formed in collaboration with M. Bauer, K. Rossnagel and coworkers (Christian-Albrechts-Universität zu Kiel, Germany) using XUV femtosecond pulses allowed to compare the dynamics at the $\Gamma$ - and $M$-points [57]. We observed very similar dynamic spectral weight redistribution at the BZ boundary and center, which corroborated the conclusion that the coherent response originates from a periodic change of the chemical potential, contrary to changes of a particular electronic state as observed earlier for several other materials [58, 25,59]. This effect was explained by an inhibited restoration of the equilibrium chemical potential after the optical excitation due to the rather small electron diffusivity perpendicular to the FeAs-layers in consequence of the small Fermi velocity $v_{\mathrm{F}, \perp}$ such that the modulation of the chemical potential by the coherent phonon persists until 2 ps [57].

4.2 Analysis of e-ph coupling Now we return to the incoherent dynamics and consider the transient broadening of the electron distribution, see Fig. 13. An analysis of the femtosecond dynamics by a heat bath model derived from a two-temperature model in which the electronic excitation is accounted for by a change in the electron temperature $T_{\mathrm{e}}$ and subsequent energy transfer represented by an increase in phonon / lattice temperatures $T_{\mathrm{ph}}, T_{1}$ was proposed by Allen [60]. It was with some modification applied successfully to various materials to describe the content of excess energy in agreement with trARPES observations [24, 52,61]. In order to verify this approach we compared in Ref. [55] three different ways to determine the electronphonon coupling strength $\lambda\left\langle\omega^{2}\right\rangle$.

Fig. 14 depicts in the left panel trARPES spectra for different delays in the vicinity of $E_{\mathrm{F}}$ on a logarithmic intensity scale. With increasing pump-probe delay the deviations between a thermalized distribution function and the measured spectrum recedes. It takes, however, about $0.5 \mathrm{ps}$ until a thermalized electron distribution function is encountered in the data. This might question the Allen approach conceptually on the one hand side. On the other hand side the deviations of the distribution function from a thermalized one occur above $100-200 \mathrm{meV}$ above $E_{\mathrm{F}}$, which is above phonon energies in the Fe-pnictides. Therefore we compare the result of such a heat bath model with further methods to analyze energy transfer to phonons. We start the discussion with determination of the electron temperature by fitting it to the trARPES spectra at different time delays and plot the result in the right panel of Fig. 14. The obtained time-dependent temperature exhibits a generic behavior. The initial maximum in temperature can be assigned to $T_{\mathrm{e}}$ and originates from the low specific heat of the electron system. Subsequently, the electron temperature reduces due to energy transfer to phonons. The transient behavior of the time evolution of temperature requires to consider two subsets of phonons, a strongly coupled hot mode and the remaining modes which might represent acoustic excitations. For the detailed model see Ref. [55]. Fig. 14 shows at right that the overall behavior of the temperature
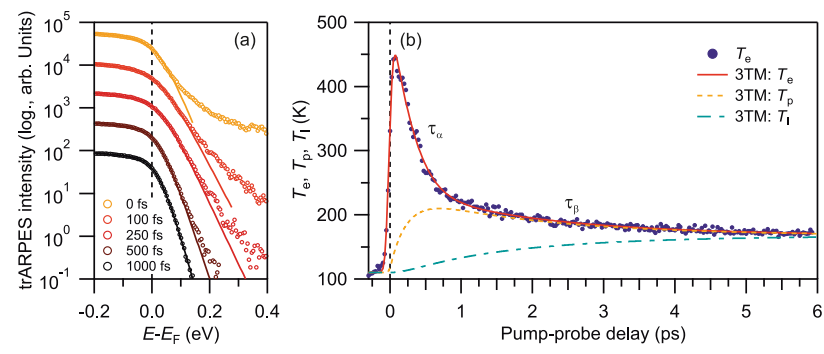

Figure 14 (a) trARPES spectra of $\mathrm{EuFe}_{2} \mathrm{As}_{2}$ at $T=100 \mathrm{~K}$ and at normal emission for various pump-probe delays on a logarithmic intensity scale using an incident pump fluence of $F=0.8 \mathrm{~mJ} \mathrm{~cm}^{-2}$. Lines are fits to Fermi-Dirac distribution functions. (b) Electronic temperature $T_{\mathrm{e}}$ determined from the fitting shown in panel (a) and a fit to the three temperature model. Transient temperatures of the hot phonons $\left(T_{\mathrm{p}}\right)$ and the rest of the lattice modes $\left(T_{1}\right)$ are shown as dashed and dash-dotted lines, respectively. Reprinted with permission from [55]. Copyright 2013 by IOP Publishing and Deutsche Physikalische Gesellschaft.

is reproduced well by such a three-temperature model. The energy transfer from electrons to the hot phonons is determined by the Eliashberg e-ph coupling function $\lambda\left\langle\omega^{2}\right\rangle$ times the temperature difference between the electron and hot phonon heat baths. Since the temperatures are known, $\lambda\left\langle\omega^{2}\right\rangle$ was determined and given in the left column of table 1 . Note that there is an increasing trend for the different samples investigated from parent to Co-doped $\mathrm{BaFe}_{2} \mathrm{As}_{2}$ to $\mathrm{EuFe}_{2} \mathrm{As}_{2}$.

In a second method to determine e-ph coupling the electron excess energy is analyzed and an assumption of a thermalized electron gas is avoided. Thermalized and nonthermalized electrons contribute to this energy according to their quasiparticle energy above $E_{\mathrm{F}}$. In this analysis the decay rates of electrons is assumed to follow the quadratic energy dependence of the Fermi liquid theory. Very close to $E_{\mathrm{F}}$ this becomes a negligible contribution because scattering with phonons is dominant for an Einstein mode above its frequency $\omega_{0}$. Fig. 15 (a) shows these two scattering rates in comparison and a dominant e-ph scattering rate up to $300 \mathrm{meV}$. The rate of energy dissipation of an electron due to emission of a phonon with energy frequency $\omega_{0}$ can then be approximated by

$$
\frac{\mathrm{d} E}{\mathrm{~d} t}=\frac{\hbar \omega_{0}}{\tau}=\pi \hbar \lambda \omega_{0}^{2}
$$

and linear in time relaxation of electron energy. The rate of energy relaxation of hot electrons has been extracted from the experimental trARPES intensity $I(E, t)$ by analyzing the mean excess energy by multiplying the intensity at $E, t$ with the electron energy, for details see Ref. [55]. Fig. 15 (b) give the experimental results for the mean excess energy determined within the energy interval highlighted in blue in Fig. 15. a). The expected linear time dependence 


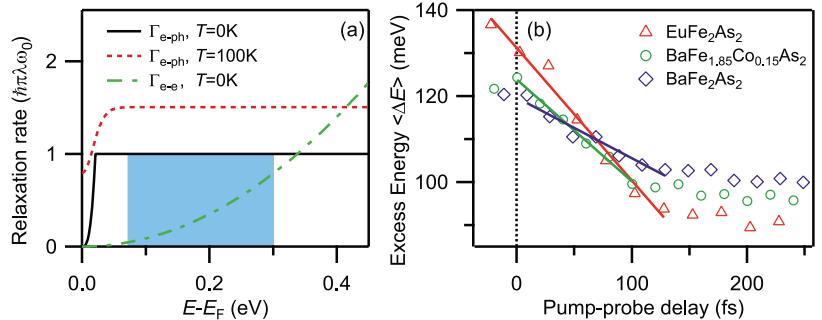

Figure 15 (a) e-ph and e-e contributions to the electron decay rate $\Gamma$. The e-ph contribution $\Gamma_{e-p h}$ calculated in the Debye model with $\hbar \omega_{\mathrm{D}}=20 \mathrm{meV}$ and $\lambda=0.3$ at $T=0$ and $100 \mathrm{~K}$ increases up to $\hbar \omega_{\mathrm{D}}$ and is constant above. The electronic contribution $\Gamma_{e-e}$ calculated for $\beta=0.1 \mathrm{eV}^{-1}$ exceeds $\Gamma_{e-p h}$ only at higher energies above $0.34 \mathrm{eV}$. The shaded area marks the energy where e-ph scattering dominates. (b) Mean electron excess energy extracted from trARPES data at $T=100 \mathrm{~K}$ near $k_{\mathrm{F}}$ within the energy window $0.07 \mathrm{eV}<\varepsilon<0.3 \mathrm{eV}$ marked in (a). Solid lines are fits, see text. Reprinted with permission from [55]. Copyright 2013 by IOP Publishing and Deutsche Physikalische Gesellschaft.

Table 1 Values of $\lambda\left\langle\omega^{2}\right\rangle$ determined by three different methods described in the main text.

\begin{tabular}{l|l|l|l}
\hline compound & $T_{e}(t)$ & $\langle\Delta E(t)\rangle$ & $\tau^{-}$ \\
\hline $\mathrm{EuFe}_{2} \mathrm{As}_{2}$ & $56-65$ & $65(5)$ & $90(30)$ \\
$\mathrm{BaFe}_{1.85} \mathrm{Co}_{0.15} \mathrm{As}_{2}$ & $46-55$ & $50(3)$ & - \\
$\mathrm{BaFe}_{2} \mathrm{As}_{2}$ & $30-46$ & $34(6)$ & - \\
\hline
\end{tabular}

is indeed obtained and the slope allows to determine $\lambda\left\langle\omega^{2}\right\rangle$ directly. The respective results is added to Tab. 1 , center column, and is within the error bar in good agreement with the results obtained from the heat bath model for all three investigated compounds.

A further way to analyze electron-phonon coupling is to analyze the dependence of the relaxation time as a function of static temperature. Within the limit of similar e-e and e-ph scattering rates, which is a reasonable assumption close to $E_{\mathrm{F}}$, see Fig. 15. (a), Kabanov and Alexandrov derived an expression for the measured relaxation time which depends linearly on $T / \lambda\left\langle\omega^{2}\right\rangle$. As is discussed in the next section, the measured relaxation time depends on the electron momentum and we have identified the relaxation time of photo-excited holes $\tau^{-}$to be independent on the spin density wave formation below $T_{\mathrm{N}}$. The observed temperature dependence is shown in Fig. 17] and follows a linear temperature dependence above $70 \mathrm{~K}$. Such temperature dependent data were obtained for $\mathrm{EuFe}_{2} \mathrm{As}_{2}$ and the resulting value for $\lambda\left\langle\omega^{2}\right\rangle$ agrees within the error bar with the results of the other analysis. We therefore conclude that the determination of $\lambda\left\langle\omega^{2}\right\rangle$ is robust. These results agree reasonably well with other experimental reports [62,63].
We estimate a value of $\lambda$ for a given $\omega$. For the Raman active $A_{1 \mathrm{~g}}$ mode at $23 \mathrm{meV}$ which is photo-excited, see Fig. 13, we find $\lambda<0.2$ for all investigated compounds. This finding is in agreement with calculations using density functional theory, which report values of $\lambda<0.35$ [64] 65]. The determined $\lambda\left\langle\omega^{2}\right\rangle$ gives for the lowest coupled modes of $12 \mathrm{meV}$ a value of 0.5 . All together these results suggest limited importance of direct e-ph coupling for the pairing mechanism in these materials. However, a cooperative interaction like magneto-phonon coupling proposed due to the dependence of the superconducting critical temperature on the pnictogen height [2] could lead to an effective enhancement of such a weak e-ph coupling. Taking into account absolute information of the $\mathrm{A}_{1 \mathrm{~g}}$ vibrational amplitude and direction obtained in time-resolved hard $\mathrm{x}$ ray diffraction changes of the magnetic moment by $11 \%$ were concluded 66. In case of such a cooperative coupling mechanism e-ph coupling at such low $\lambda$ values are to be considered as a prerequisite for superconductivity.

4.3 Spin-dependent relaxation Besides coupling to phonons optically excited electrons can present spindependent relaxation. In the strongly correlated case of cuprates the time scales are in the range of 1-10 fs and therefore likely below the sensitivity of the present femtosecond photoemission experiment. However, in the antiferrimagnetically ordered phase of parent $\mathrm{EuFe}_{2} \mathrm{As}_{2}$ we observed considerably slower spin-dependent relaxation employing momentum-resolved detection in trARPES. Fig. 16(a) depicts the pump-induced ARPES intensity change $100 \mathrm{fs}$ after the optical excitation. In the BZ center we observe a clear intensity increase at energies above $E_{\mathrm{F}}$, while at $k>k_{\mathrm{F}}$ the intensity decrease dominates below $E_{\mathrm{F}}$. One might expect such a momentum-dependent intensity change due to electron and hole excitation within the Fe $3 \mathrm{~d}$ bands forming the hole pockets centered at $\Gamma$. However, the analysis of the transient spectral weight redistribution shows that such a single electron picture only holds at $T>T_{\mathrm{N}}$. In Figs. 16. b,c) spectra after optical pumping are compared for different $k$ and $T$. At $210 \mathrm{~K}>T_{\mathrm{N}}$ intensity decrease and increase are observed symmetrically above and below $E_{\mathrm{F}}$, respectively. This can be understood in a single particle picture and an increased $T_{\mathrm{e}}$ after laser excitation, see Sec. 4.2. However, for $T<T_{\mathrm{N}}$ the spectral redistribution becomes momentum dependent. While at $k>k_{\mathrm{F}}$ the symmetric intensity redistribution persists, at $\Gamma$ we observe a spectral weight shift to higher energy and almost exclusively intensity increase. Considering the backfolding and AFM energy gap in the electronic structure in the antiferromagnetic phase, see Fig.12.c) allows to understand this dynamic behavior. Upon photoexcitation, the gap closes and is filled by electrons, evidenced by the shift of the leading edge and the strong increase of spectral weight at $E_{\mathrm{F}}$.

The difference in dynamic spectral weight redistribution has an interesting counterpart in the relaxation times, which were analyzed by integrating the intensity in the en- 

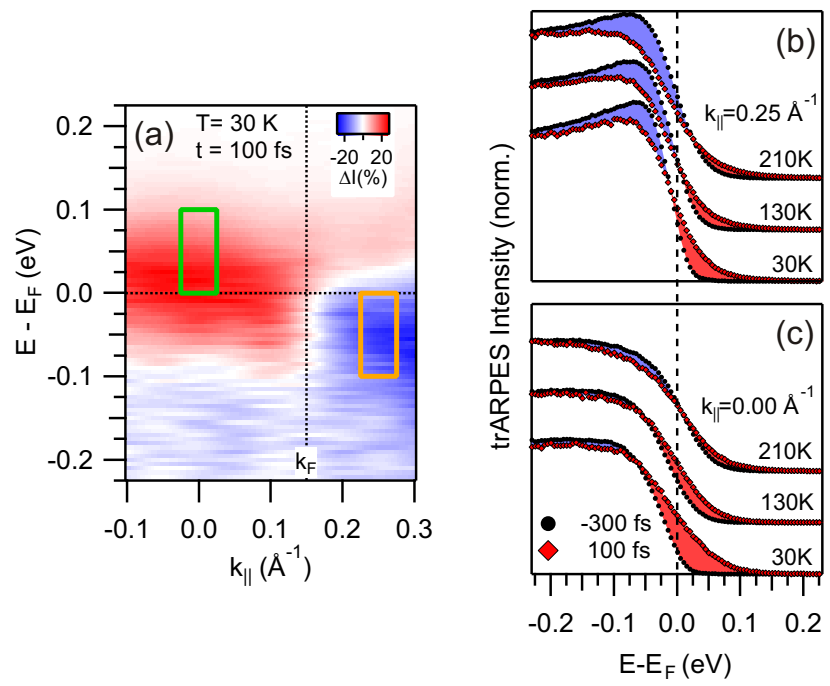

Figure 16 (a) Pump-induced change of spectral weight $\delta I$ in a false-color map for $\Delta t=100 \mathrm{fs}$. Blue color marks depletion of spectral weight (hole excitations), while red marks increased $\delta I$ (electron excitations). Boxes represent integration areas for $\delta I$. (b) Energy distribution curves (EDCs) for $k_{\|}=0.25 \AA^{-1}>k_{\mathrm{F}}$ before (black solid circles) and $t=100 \mathrm{fs}$ after laser excitation (red diamonds) for various temperatures. Increase and depletion of spectral weight are marked by red and blue areas, respectively. Spectra are vertically offset for clarity. (c) EDCs for $k_{\|}=0$. Reprinted with permission from [54]. Copyright 2012 by the American Physical Society.

ergy and momentum intervals indicated in Fig. 16.a) in green and orange, see [54] for details. At the lowest $T$ the relaxation times at the two selected momenta differ more than a factor 4 and the relaxation near $k_{\mathrm{F}}$ is faster than around $\Gamma$ while they coincide above $T_{\mathrm{N}}$. In fact, the temperature-dependent relaxation times resemble the temperature dependence of the antiferromagnetic order parameter, which is straightforward to understand. Relaxation near $\Gamma$ requires that the electrons relax in a spin-dependent manner such that they match or even reestablish antiferromagnetic order. This lowers the relaxation phase space for such electrons, which slows down the relaxation time. Electrons near $k_{\mathrm{F}}$ do not contribute to antiferromagnetic order and relax by energy transfer to phonons, which we have exploited already in the previous section in the analysis of e-ph coupling. In conclusion, we have succeeded to determine a considerable more than four time difference in the relaxation time of spin-dependent dynamics in the antiferromagnetic over the paramagnetic state. Further the influence of the structural change from tetragonal to orthorhombic and nematic fluctuations plays a role in the detailed time-dependent spectral weight transfer, see Ref. [54], further insight might profit from increased statistics and further optimized time-bandwidth product of the employed UV laser pulses.

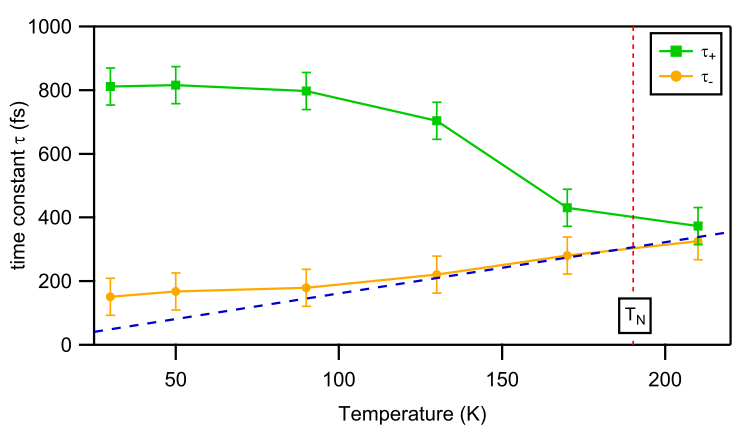

Figure 17 Temperature dependent relaxation times $\tau^{+}, \tau^{-}$ of the spectral weight increase at $\Gamma$ and decrease at $k_{\mathrm{F}}$, respectively, published in Ref. [54] compared to a model prediction with a linearly increasing relaxation time as a function of temperature.

5 Conclusion The collaborative activity of combining static and femtosecond time-resolved ARPES turned out to be very fruitful in terms of complementary information obtained in the spectral and time domains. Essential for better understanding what is and what is not measured by these two techniques is to appreciate the actual observables in static and time-resolved ARPES. While the first method probes single particle excitations, the latter one analyzes relaxation and energy dissipation of optically excited populations of single to many particles. In consequence, the relaxation rates determined by these two methods can differ significantly [49,67].

In the present study, both techniques indicate that the coupling of the charge carriers to phonons is considerably weaker than the coupling to electronic degrees of freedom. In particular the results from static ARPES point to the importance of electronic correlation effects, a result which can be derived from the NFL behavior of the scattering rates. In combination with the detected Lifshitz transition near optimally doping the results offer an explanation for the strange normal state properties, which may be different from the widely discussed quantum critical scenario. In the superconducting state, the results point to an interpolation state between BCS and BE condensation.

Acknowledgements This work was supported by the German Research Foundation, the DFG, through the priority program SPP 1458.

\section{References}

[1] Y. Kamihara, T. Watanabe, M. Hirano, and H. Hosono, J. Am. Chem. Soc. 130, 3296 (2008).

[2] D. C. Johnston, Adv. Phys. 59, 803 (2010).

[3] A. A. Kordyuk, Low Temp. Phys. 38, 888 (2012).

[4] H. v. Löhneysen, A. Rosch, M. Vojta, and P. Wölfle, Rev. Mod. Phys. 79, 1015 (2007).

[5] P. Gegenwart, Q. Si, and F. Steglich, Nat. Phys. 4, 186 (2008). 
[6] R. Haslinger, A. Abanov, and A. Chubukov, Europhys. Lett. 58, 271 (2002).

[7] S. Kasahara, T. Shibauchi, K. Hashimoto, K. Ikada, S. Tonegawa, R. Okazaki, H. Shishido, H. Ikeda, H. Takeya, K. Hirata, T. Terashima, and Y. Matsuda, Phys. Rev. B 81, 184519 (2010).

[8] J. G. Analytis, H. H. Kuo, R. D. McDonald, M. Wartenbe, P. M. C. Rourke, N. E. Hussey, and I. R. Fisher, Nat. Phys. 10, 194 (2014).

[9] C. Varma, Z. Nussinov, and W. van Saarloos, Phys. Rep. 361, 267 (2002).

[10] F. Rullier-Albenque, Comptes Rendus Physique 17, 164 (2016).

[11] I. I. Mazin, D. J. Singh, M. D. Johannes, and M. H. Du, Phys. Rev. Lett. 101, 057003 (2008).

[12] A. Damascelli, Z. Hussain, and Z.X. Shen, Rev. Mod. Phys. 75, 473 (2003).

[13] A. F. Kemper, M. M. Korshunov, T. P. Devereaux, J. N. Fry, H. P. Cheng, and P. J. Hirschfeld, Phys. Rev. B 83, 184516 (2011).

[14] S. Thirupathaiah, PhD thesis, TU Berlin, 2011.

[15] F. Massee, Y. Huang, R. Huisman, S. de Jong, J. B. Goedkoop, and M. S. Golden, Phys. Rev. B 79, 220517(R) (2009).

[16] F. Hardy, P. Adelmann, T. Wolf, H. v. Löhneysen, and C. Meingast, Phys. Rev. Lett. 102, 187004 (2009), and A. Böhmer et al. (unpublished).

[17] H. S. Jeevan, D. Kasinathan, H. Rosner, and P. Gegenwart, Phys. Rev. B 83, 054511 (2011).

[18] F. Steckel, M. Roslova, R. Beck, I. Morozov, S. Aswartham, D. Evtushinsky, C. G. F. Blum, M. AbdelHafiez, D. Bombor, J. Maletz, S. Borisenko, A. V. Shevelkov, A. U.B. Wolter, C. Hess, S. Wurmehl, and B. Büchner, Phys. Rev. B 91, 184516 (2015).

[19] S. Thirupathaiah, S. de Jong, R. Ovsyannikov, H. A. Dürr, A. Varykhalov, R. Follath, Y. Huang, R. Huisman, M. S. Golden, Y.Z. Zhang, H. O. Jeschke, R. Valenti, A. Erb, A. Gloskovskii, and J. Fink, Phys. Rev. B 81, 104512 (2010).

[20] J. Fink, S. Thirupathaiah, R. Ovsyannikov, H. A. Duerr, R. Follath, Y. Huang, S. de Jong, M. S. Golden, Y.Z. Zhang, H. O. Jeschke, R. Valenti, C. Felser, S. D. Farahani, M. Rotter, and D. Johrendt, Phys. Rev. B 79, 155118 (2009).

[21] S. de Jong, E. van Heumen, S. Thirupathaiah, R. Huisman, F. Massee, J. B. Goedkoop, R. Ovsyannikov, J. Fink, H. A. Dürr, A. Gloskovskii, H. S. Jeevan, P. Gegenwart, A. Erb, L. Patthey, M. Shi, R. Follath, A. Varykhalov, and M. S. Golden, EPL 89, 27007 (2010).

[22] E. van Heumen, J. Vuorinen, K. Koepernik, F. Massee, Y. Huang, M. Shi, J. Klei, J. Goedkoop, M. Lindroos, J. van den Brink, and M. S. Golden, Phys. Rev. Lett. 106, 027002 (2011)

[23] A. Lankau, K. Koepernik, S. Borisenko, V. Zabolotnyy, B. Büchner, J. van den Brink, and H. Eschrig, Phys. Rev. B 82, 184518 (2010).

[24] M. Lisowski, P. Loukakos, U. Bovensiepen et al., Appl. Phys. A 78, 165 (2004).

[25] F. Schmitt, P. Kirchmann, U. Bovensiepen, R. G. Moore, J. H. Chu, D. H. Lu, L. Rettig, M. Wolf, I. R. Fisher, and Z.-X. Shen, New J. Phys. 13, 063022 (2011).
[26] S. Thirupathaiah, E. D. L. Rienks, H. S. Jeevan, R. Ovsyannikov, E. Slooten, J. Kaas, E. van Heumen, S. de Jong, H. A. Dürr, K. Siemensmeyer, R. Follath, P. Gegenwart, M. S. Golden, and J. Fink, Phys. Rev. B 84, 014531 (2011).

[27] A. Herbig, R. Heid, J. Schmalian, arXiv: 1510.06941.

[28] I. M. Lifshitz, Sov. Phys. JETP 11, 1130 (1960).

[29] J. Fink, A. Charnukha, E. D. L. Rienks, Z. H. Liu, S. Thirupathaiah, I. Avigo, F. Roth, H. S. Jeevan, P. Gegenwart, M. Roslova, I. Morozov, S. Wurmehl, U. Bovensiepen, S. Borisenko, M. Vojta, and B. Büchner, Phys. Rev. B 92, 201106 (2015).

[30] E. D. L. Rienks, T. Wolf, K. Koepernik, I. Avigo, P. Hlawenka, C. Lupulescu, T. Arion, F. Roth, W. Eberhardt, U. Bovensiepen, and J. Fink, EPL (Europhysics Letters) 103, 47004 (2013).

[31] S. Engelsberg and J.R. Schrieffer, Phys. Rev. 131, 993 (1963).

[32] G. D. Mahan, Many-Particle Physics (Kluwer Academic/Plenum Publishers, New York, 2000).

[33] S. Graser, T. A. Maier, P. J. Hirschfeld, and D. J. Scalapino, New J. Phys. 11, 025016 (2009).

[34] J. Fink, EPL (Europhysics Letters) 113, 27002 (2016).

[35] J. Fink, unpublished.

[36] K. Hashimoto, K. Cho, T. Shibauchi, S. Kasahara, Y. Mizukami, R. Katsumata, Y. Tsuruhara, T. Terashima, H. Ikeda, M. A. Tanatar, H. Kitano, N. Salovich, R. W. Giannetta, P. Walmsley, A. Carrington, R. Prozorov, and Y. Matsuda, Science 336, 1554 (2012).

[37] P. Walmsley, C. Putzke, L. Malone, I. Guillamn, D. Vignolles, C. Proust, S. Badoux, A. I. Coldea, M. D. Watson, S. Kasahara, Y. Mizukami, T. Shibauchi, Y. Matsuda, and A. Carrington, Phys. Rev. Lett. 110, 257002 (2013).

[38] H. Shishido, A. F. Bangura, A. I. Coldea, S. Tonegawa, K. Hashimoto, S. Kasahara, P. M. C. Rourke, H. Ikeda, T. Terashima, R. Settai, Y. Ōnuki, D. Vignolles, C. Proust, B. Vignolle, A. McCollam, Y. Matsuda, T. Shibauchi, and A. Carrington, Phys. Rev. Lett. 104, 057008 (2010).

[39] S. Arsenijevic, H. Hodovanets, R. Gal, L. Forr, S. L. Bud'ko, and P.C. Canfield, Phys. Rev. B 87, 224508 (2013).

[40] C. Meingast, F. Hardy, R. Heid, P. Adelmann, A. Böhmer, P. Burger, D. Ernst, R. Fromknecht, P. Schweiss, and T. Wolf, Phys. Rev. Lett. 108, 177004 (2012).

[41] J. Labbe and J. Friedel, J. Phys. France 27, 153 (1966).

[42] Y. J. Uemura, L. P. Le, G. M. Luke, B. J. Sternlieb, W. D. Wu, J.H. Brewer, T.M. Riseman, C.L. Seaman, M. B. Maple, M. Ishikawa, D. G. Hinks, J. D. Jorgensen, G. Saito, and H. Yamochi, Phys. Rev. Lett. 66, 2665 (1991).

[43] A. B. Migdal, Soviet Physics JETP - USSR 34(6), 9961001 (1958)

[44] J. Bardeen, L. N. Cooper, and J. R. Schrieffer, Phys. Rev. 108, 1175 (1957).

[45] C. Grimaldi, L. Pietronero, and S. Strässler, Phys. Rev. Lett. 75, 1158 (1995).

[46] S. Borisenko, Nature Mater. 12, 600 (2013).

[47] S. N. Bose, Z. Phys 26, 178 (1924).

[48] U. Bovensiepen, H. Petek, and M. Wolf, Dynamics in Solid States Surfaces and Interfaces, Vol. 1, Current Developments (Wiley-VCH, Weinheim, 2010). 
[49] S. L. Yang, J. A. Sobota, D. Leuenberger, Y. He, M. Hashimoto, D. H. Lu, H. Eisaki, P. S. Kirchmann, and Z. X. Shen, Phys. Rev. Lett. 114, 247001 (2015).

[50] R. D. Averitt and A. J. Taylor, J. Phys: Condens. Matter 14, 1357(R) (2002).

[51] W. Nessler, S. Ogawa, H. Nagano, H. Petek, J. Shimoyama, Y. Nakayama, and K. Kishio, Phys. Rev. Lett. 81, 4480 (1998).

[52] L. Perfetti, P. A. Loukakos, M. Lisowski, U. Bovensiepen, H. Eisaki, and M. Wolf, Phys. Rev. Lett. 99, 197001 (2007).

[53] U. Bovensiepen and P. S. Kirchmann, Laser \& Photonics Reviews 6, 589 (2012).

[54] L. Rettig, R. Cortés, S. Thirupathaiah, P. Gegenwart, H. S. Jeevan, M. Wolf, J. Fink, and U. Bovensiepen, Phys. Rev. Lett. 108, 097002 (2012).

[55] L. Rettig, R. Cortes, H. S. Jeevan, P. Gegenwart, T. Wolf, J. Fink, and U. Bovensiepen, New J. Phys. 15, 083023 (2013).

[56] I. Avigo, R. Cortés, L. Rettig, S. Thirupathaiah, H. S. Jeevan, P. Gegenwart, T. Wolf, M. Ligges, M. Wolf, J. Fink, and U. Bovensiepen, J. Phys.:Condens. Matter 25, 094003 (2013).

[57] L. X. Yang, G. Rohde, T. Rohwer, A. Stange, K. Hanff, C. Sohrt, L. Rettig, R. Cortés, F. Chen, D. L. Feng, T. Wolf, B. Kamble, I. Eremin, T. Popmintchev, M. M. Murnane, H. C. Kapteyn, L. Kipp, J. Fink, M. Bauer, U. Bovensiepen, and K. Rossnagel, Phys. Rev. Lett. 112, 207001 (2014).

[58] L. Perfetti, P. A. Loukakos, M. Lisowski, U. Bovensiepen, H. Berger, S. Biermann, P. S. Cornaglia, A. Georges, and M. Wolf, Phy. Rev. Lett. 97, 067402 (2006).

[59] S. Hellmann, T. Rohwer, M. Kalläne, K. Hanff, C. Sohrt, a. Stange, a. Carr, M. Murnane, H. Kapteyn, L. Kipp, M. Bauer, and K. Rossnagel, Nature Communications 3, 1069 (2012).

[60] P. B. Allen, Phys. Rev. Lett. 59, 1460-1463 (1987).

[61] U. Bovensiepen, Journal of Physics: Condensed Matter 19, 083201 (2007).

[62] B. Mansart, D. Boschetto, A. Savoia, F. Rullier-Albenque, F. Bouquet, E. Papalazarou, A. Forget, D. Colson, A. Rousse, and M. Marsi, Phys. Rev. B 82, 024513 (2010).

[63] L. Stojchevska, P. Kusar, T. Mertelj, V. V. Kabanov, X. Lin, G. H. Cao, Z. A. Xu, and D. Mihailovic, Phys. Rev. B 82, 012505 (2010).

[64] L. Boeri, O. V. Dolgov, and A. A. Golubov, Phys. Rev. Lett. 101, 026403 (2008).

[65] L. Boeri, M. Calandra, I. I. Mazin, O. V. Dolgov, and F. Mauri, Phys. Rev. B 82, 020506 (2010).

[66] L. Rettig, S. O. Mariager, A. Ferrer, S. Grübel, J. A. Johnson, J. Rittmann, T. Wolf, S. L. Johnson, G. Ingold, P. Beaud, and U. Staub, Phys. Rev. Lett. 114, 067402 (2015).

[67] I. Avigo, S. Thirupathaiah, M. Ligges, T. Wolf, J. Fink, and U. Bovensiepen, arXiv: 1605.05177. 